\title{
Tutorial on Photoacoustic Microscopy and Computed Tomography
}

\author{
Lihong V. Wang \\ (Invited Paper)
}

\begin{abstract}
The field of photoacoustic tomography has experienced considerable growth in the past few years. Although several commercially available pure optical imaging modalities, including confocal microscopy, two-photon microscopy, and optical coherence tomography, have been highly successful, none of these technologies can provide penetration beyond $\sim 1 \mathrm{~mm}$ into scattering biological tissues, because they are based on ballistic and quasi-ballistic photons. Heretofore, there has been a void in highresolution optical imaging beyond this penetration limit. Photoacoustic tomography, which combines high ultrasonic resolution and strong optical contrast in a single modality, has broken through this limitation and filled this void. In this paper, the fundamentals of photoacoustics are first introduced. Then, scanning photoacoustic microscopy and reconstruction-based photoacoustic tomography (or photoacoustic computed tomography) are covered.
\end{abstract}

Index Terms-Microwave-induced acoustic imaging, optical imaging, optoacoustic imaging, photoacoustic imaging (PAI), photoacoustic microscopy (PAM), photoacoustic tomography, thermoacoustic tomography.

\section{INTRODUCTION}

$\mathbf{P}$ HOTOACOUSTIC IMAGING (PAI) refers to imaging that is based on the photoacoustic effect. Although the photoacoustic effect as a physical phenomenon was first reported by Alexander Graham Bell in 1880 [1], PAI as an imaging technology was developed only after the advent of ultrasonic transducers, computers, and lasers [2]-[31]. In this paper, we will focus on the fundamentals of photoacoustics, which is adapted from a chapter in [32], then illustrate PAI with two implementations. A comprehensive review on biomedical photoacoustics and imaging is available elsewhere [33].

The motivation for PAI is to combine optical-absorption contrast with ultrasonic spatial resolution for deep imaging in the optical quasi-diffusive or diffusive regime, which is $>1 \mathrm{~mm}$ in most biological tissues. In PAI, the tissue is irradiated usually by a short-pulsed laser beam to achieve a thermal and acoustic impulse response. Locally absorbed light is converted into heat, which is further converted to a pressure rise via thermoelastic expansion. The initial pressure rise-determined by the local optical energy deposition (specific or volumetric optical absorp-

Manuscript received September 10, 2007; revised November 12, 2007. This work was supported by the National Institutes of Health under Grant R01 EB000712 and Grant R01 NS46214.

The author is with the Optical Imaging Laboratory, Department of Biomedical Engineering, Washington University, St. Louis, MO 63130-4899 USA (e-mail: lhwang@biomed.wustl.edu).

Color versions of one or more of the figures in this paper are available online at http://ieeexplore.ieee.org.

Digital Object Identifier 10.1109/JSTQE.2007.913398 tion) and other thermal and mechanical properties-propagates as an ultrasonic wave, which is referred to as a photoacoustic wave. The photoacoustic wave is detected by an ultrasonic transducer, producing an electric signal. The electric signal is then amplified, digitized, and transferred to a computer. To form an image, a single-element ultrasonic transducer is scanned around the tissue; alternatively, an ultrasonic array can be used to acquire data in parallel.

PAI has two major forms of implementation. One is based on a scanning-focused ultrasonic transducer. Dark-field confocal photoacoustic microscopy belongs to this category. The other is based on an array of unfocused ultrasonic transducers in combination with a reconstruction algorithm. A circular detection configuration will be illustrated.

The image contrast of PAI is based on optical absorption in the photoacoustic excitation phase. Selective optical absorption is associated with molecules such as oxygenated and deoxygenated hemoglobin and melanin. Concentrations of multiple chromophores whose spectra of absorption coefficient are different can be quantified simultaneously by varying the wavelength of the irradiating laser. Such quantification of oxygenated and deoxygenated hemoglobin, for example, can provide functional imaging of the total concentration and oxygen saturation of hemoglobin.

The spatial resolution of PAI is derived from ultrasonic detection in the photoacoustic emission phase. Due to strong optical scattering, pure optical imaging in biological tissue has either shallow imaging depth or low spatial resolution. Although light scattering broadens and attenuates light reaching an embedded object, the resulting ultrasonic signal provides better resolution than the optical signal in the optical quasi-diffusive or diffusive regime, because ultrasonic scattering is two to three orders of magnitude weaker than optical scattering. The image resolution, as well as the maximum imaging depth, is scaleable with the ultrasonic frequency within the reach of diffuse photons. Specifically, as the ultrasonic center frequency and bandwidth increase, the spatial resolution improves at the expense of imaging depth, because ultrasonic attenuation increases with frequency. In addition, PAI provides images devoid of speckle artifacts, which are conspicuous in images acquired with either ultrasonography or optical coherence tomography.

\section{Initial Photoacoustic Pressure}

Upon short laser pulse excitation, local fractional volume expansion $d V / V$ of the heated tissue at position $\mathbf{r}$ can be 
expressed as

$$
\frac{d V}{V}=-\kappa p(\mathbf{r})+\beta T(\mathbf{r}) .
$$

Here, $\kappa$ denotes the isothermal compressibility $(\sim 5 \times$ $10^{-10} \mathrm{~Pa}^{-1}$ for water or soft tissue), $\beta$ denotes the thermal coefficient of volume expansion ( $\sim \times 10^{-4} \mathrm{~K}^{-1}$ for muscle), and $p$ and $T$ denote the changes in pressure (in Pascal) and temperature (in Kelvin), respectively. The isothermal compressibility $\kappa$ can be expressed as

$$
\kappa=\frac{C_{P}}{\rho v_{s}^{2} C_{V}} .
$$

Here, $\rho$ denotes the mass density $\left(\sim 1000 \mathrm{~kg} / \mathrm{m}^{3}\right.$ for water and soft tissue), $v_{s}$ denotes the speed of sound ( $\sim 1480 \mathrm{~m} / \mathrm{s}$ in water), and $C_{P}$ and $C_{V}[\sim 4000 \mathrm{~J} /(\mathrm{kg} \cdot \mathrm{K})$ for muscle $]$ denote the specific heat capacities at constant pressure and volume, respectively. It is important to distinguish between $C_{P}$ and $C_{V}$ for gasses but not for tissue.

Here, we assume that the laser pulse duration $t_{L}$ is less than the acoustic confinement time-which is less than the thermal confinement time

$$
t_{L}<\frac{d_{c}}{v_{s}}<\frac{d_{c}^{2}}{4 \alpha_{\mathrm{th}}}
$$

where $d_{c}$ is the characteristic length of heat heterogeneity (the dimension of the optically absorbing target of interest or the decay constant of the optical energy deposition, whichever is smaller), and $\alpha_{\mathrm{th}}$ is the thermal diffusivity $\left(\sim 0.1 \mathrm{~mm}^{2} / \mathrm{s}\right.$ for tissue).

For such a short laser pulse, the fractional volume expansion is negligible and the local pressure rise $p_{0}$ immediately after the laser excitation can be derived from (1) [34] as

$$
p_{0}(\mathbf{r})=\frac{\beta T(\mathbf{r})}{\kappa} .
$$

It can be shown that each millikelvin temperature rise yields an 8 mbar (or $800 \mathrm{~Pa}$ ) pressure rise. If we assume that all absorbed optical energy is converted into heat and nonthermal relaxation such as fluorescence is negligible, the temperature rise generated by the short laser pulse is

$$
T=\frac{A_{e}}{\rho C_{V}}
$$

where $A_{e}$ is the specific or volumetric optical absorption (in joules per centimeter cubed, optical energy deposition density).

From (4) and (5), we have

$$
p_{0}=\frac{\beta}{\kappa \rho C_{V}} A_{e} .
$$

We define the Grueneisen parameter (dimensionless) as

$$
\Gamma=\frac{\beta}{\kappa \rho C_{V}} .
$$

Hence, (6) becomes

$$
p_{0}=\Gamma A_{e} .
$$

\section{Photoacoustic EQuATion}

The photoacoustic wave generation and propagation in an inviscid medium is described by the general photoacoustic equation

$$
\left(\nabla^{2}-\frac{1}{v_{s}^{2}} \frac{\partial^{2}}{\partial t^{2}}\right) p(\mathbf{r}, t)=-\frac{\beta}{\kappa v_{s}^{2}} \frac{\partial^{2} T(\mathbf{r}, t)}{\partial t^{2}}
$$

where $p(\mathbf{r}, t)$ denotes the acoustic pressure at location $\mathbf{r}$ and time $t$, and $T$ denotes the temperature rise. The left-hand side of this equation describes the wave propagation, whereas the right-hand side represents the source term.

For a short laser pulse that satisfies the condition shown in (3), the following thermal equation holds:

$$
\rho C_{V} \frac{\partial T(\mathbf{r}, t)}{\partial t}=H(\mathbf{r}, t) .
$$

Here, $H$ is the heating function defined as the thermal energy converted per unit volume and per unit time; it is related to the optical absorption coefficient $\mu_{a}$ and fluence rate $\Phi$ by $H=\mu_{a} \Phi$. As a result, we obtain the following photoacoustic equation:

$$
\left(\nabla^{2}-\frac{1}{v_{s}^{2}} \frac{\partial^{2}}{\partial t^{2}}\right) p(\mathbf{r}, t)=-\frac{\beta}{C_{P}} \frac{\partial H(\mathbf{r}, t)}{\partial t} .
$$

Because the source term is related to the first time derivative of $H$, time-invariant heating does not produce a pressure wave; only time-variant heating does.

\section{FORWARD SOLUTION}

The general photoacoustic equation shown in (9) can be solved by the Green's function approach [35]. The Green's function is defined here as the response to a spatial and temporal impulse source term

$$
\left(\nabla^{2}-\frac{1}{v_{s}^{2}} \frac{\partial^{2}}{\partial t^{2}}\right) G\left(\mathbf{r}, t ; \mathbf{r}^{\prime}, t^{\prime}\right)=-\delta\left(\mathbf{r}-\mathbf{r}^{\prime}\right) \delta\left(t-t^{\prime}\right)
$$

where $\mathbf{r}^{\prime}$ and $t^{\prime}$ denote the source location and time, respectively. In an infinite space, where no boundary exists, the Green's function is given by

$$
G\left(\mathbf{r}, t ; \mathbf{r}^{\prime}, t^{\prime}\right)=\frac{\delta\left(t-t^{\prime}-\left|\mathbf{r}-\mathbf{r}^{\prime}\right| / v_{s}\right)}{4 \pi\left|\mathbf{r}-\mathbf{r}^{\prime}\right|}
$$

which represents an impulse diverging spherical wave. The following reciprocity relation holds:

$$
G\left(\mathbf{r}, t ; \mathbf{r}^{\prime}, t^{\prime}\right)=G\left(\mathbf{r}^{\prime},-t^{\prime} ; \mathbf{r},-t\right) .
$$

To see this relationship more clearly, one observes $G(\mathbf{r}, t$; $\left.\mathbf{r}^{\prime}, 0\right)=G\left(\mathbf{r}^{\prime}, 0 ; \mathbf{r},-t\right)$ by setting $t^{\prime}=0$.

Applying the Green's function approach to (9), we get

$$
p(\mathbf{r}, t)=\int_{-\infty}^{t^{+}} d t^{\prime} \int d \mathbf{r}^{\prime} G\left(\mathbf{r}, t ; \mathbf{r}^{\prime}, t^{\prime}\right) \frac{\beta}{\kappa v_{s}^{2}} \frac{\partial^{2} T\left(\mathbf{r}^{\prime}, t^{\prime}\right)}{\partial t^{\prime 2}}
$$

which represents the pressure in response to an arbitrary source. Substituting (13) into (15) leads to

$$
p(\mathbf{r}, t)=\left.\frac{\beta}{4 \pi \kappa v_{s}^{2}} \int d \mathbf{r}^{\prime} \frac{1}{\left|\mathbf{r}-\mathbf{r}^{\prime}\right|} \frac{\partial^{2} T\left(\mathbf{r}^{\prime}, t^{\prime}\right)}{\partial t^{\prime 2}}\right|_{t^{\prime}=t-\frac{\left|\mathbf{r}-\mathbf{r}^{\prime}\right|}{v_{s}}} .
$$


In thermal confinement, substituting (10) into (16), we get

$$
p(\mathbf{r}, t)=\left.\frac{\beta}{4 \pi C_{P}} \int d \mathbf{r}^{\prime} \frac{1}{\left|\mathbf{r}-\mathbf{r}^{\prime}\right|} \frac{\partial H\left(\mathbf{r}^{\prime}, t^{\prime}\right)}{\partial t^{\prime}}\right|_{t^{\prime}=t-\frac{\left|\mathbf{r}-\mathbf{r}_{s}\right|}{v_{s}}}
$$

or

$$
p(\mathbf{r}, t)=\frac{\beta}{4 \pi C_{P}} \frac{\partial}{\partial t} \int d \mathbf{r}^{\prime} \frac{1}{\left|\mathbf{r}-\mathbf{r}^{\prime}\right|} H\left(\mathbf{r}^{\prime}, t-\frac{\left|\mathbf{r}-\mathbf{r}^{\prime}\right|}{v_{s}}\right) .
$$

If the heating function can be decomposed as $H\left(\mathbf{r}^{\prime}, t^{\prime}\right)=$ $A_{e}\left(\mathbf{r}^{\prime}\right) H_{t}\left(t^{\prime}\right)$, where $\int H_{t}\left(t^{\prime}\right) d t^{\prime}=1$, (18) can be further simplified to

$$
p(\mathbf{r}, t)=\frac{\beta}{4 \pi C_{P}} \frac{\partial}{\partial t} \int d \mathbf{r}^{\prime} \frac{A_{e}\left(\mathbf{r}^{\prime}\right)}{\left|\mathbf{r}-\mathbf{r}^{\prime}\right|} H_{t}\left(t-\frac{\left|\mathbf{r}-\mathbf{r}^{\prime}\right|}{v_{s}}\right) .
$$

If the condition in (3) holds, we have approximately $H_{t}\left(t^{\prime}\right)=$ $\delta\left(t^{\prime}\right)$. In this case, (19) becomes

$$
p(\mathbf{r}, t)=\frac{\partial}{\partial t}\left[\frac{\beta}{4 \pi C_{P}} \frac{1}{v_{s} t} \int d \mathbf{r}^{\prime} A_{e}\left(\mathbf{r}^{\prime}\right) \delta\left(t-\frac{\left|\mathbf{r}-\mathbf{r}^{\prime}\right|}{v_{s}}\right)\right]
$$

where the quantity within the square brackets is the step-heating response of an arbitrary absorbing object and its time differentiation yields the delta-heating response. Using (7) and (8), we rewrite (20) as

$$
p(\mathbf{r}, t)=\frac{1}{4 \pi v_{s}^{2}} \frac{\partial}{\partial t}\left[\frac{1}{v_{s} t} \int d \mathbf{r}^{\prime} p_{0}\left(\mathbf{r}^{\prime}\right) \delta\left(t-\frac{\left|\mathbf{r}-\mathbf{r}^{\prime}\right|}{v_{s}}\right)\right] .
$$

This equation can be used to compute photoacoustic pressure generated by an arbitrarily heterogeneous optically absorbing object.

We now consider an optically thin slab of thickness $d$, heated up homogeneously by a delta excitation pulse. The laser beam, infinitely broad and uniform, is incident normally on the slab. The attenuation inside the slab is negligible. An initial pressure $p_{0}$ is first built up within the slab, and then, propagated outward in both the positive and the negative $z$-directions, where the $z$ axis is perpendicular to the slab with the origin on the middle plane of the slab. The initial pressure distribution can be written as

$$
p_{0}(z)=p_{0} U\left(z+\frac{d}{2}\right) U\left(-z+\frac{d}{2}\right)
$$

where $U$ is the Heaviside step function defined as

$$
U(z)=\left\{\begin{array}{l}
1 \text { for } z \geq 0 . \\
0 \text { for } z<0 .
\end{array}\right.
$$

Solving (21) leads to the pressure distribution at any later time

$$
p(z, t)=\frac{1}{2} p_{0}\left(z-v_{s} t\right)+\frac{1}{2} p_{0}\left(z+v_{s} t\right) .
$$

The first term on the right-hand side represents a right propagating (along the $+z$-axis) plane wave and the second term represents a left propagating (along the $-z$-axis) plane wave. On delta heating, pressure $p_{0}$ is generated within the slab. Then, $p_{0}$ is split into two plane waves, each having a magnitude of $p_{0} / 2$ but propagating in opposite directions.
Similarly, for a spherical object of radius $R_{s}$, we have

$$
\begin{aligned}
p(r, t)= & p_{0}\left[U\left(R_{s}-v_{s} t-r\right)+\frac{r-v_{s} t}{2 r}\right. \\
& \left.\times U\left(r-\left|R_{s}-v_{s} t\right|\right) U\left(R_{s}+v_{s} t-r\right)\right] .
\end{aligned}
$$

The radial coordinate $r$ originates at the center of the sphere. If we write the initial pressure as

$$
p_{0}(r)=p_{0} U(r) U\left(-r+R_{s}\right), \quad \text { for } 0 \leq r<R_{s}
$$

we have

$$
\begin{aligned}
p(r, t)= & \frac{r+v_{s} t}{2 r} p_{0}\left(r+v_{s} t\right)+\frac{r-v_{s} t}{2 r} p_{0}\left(-r+v_{s} t\right) \\
& +\frac{r-v_{s} t}{2 r} p_{0}\left(r-v_{s} t\right) .
\end{aligned}
$$

The first term on the right-hand side represents a converging spherical wave; the second term represents a diverging spherical wave that originates from the initially converging wave propagating through the center, and the third term represents a diverging spherical wave.

When an ultrasonic detector is placed outside the sphere at various radial locations, the detected pressures are shown in Fig. 1. Upon delta heating, an initial pressure $p_{0}$ - constant across the entire heated sphere-is generated. This initial pressure is divided into two equal parts, each initiating a spherical wave. One travels outward as a diverging spherical compression wave, yielding the first-arriving positive pressure in the plot. The other travels inward as a converging spherical compression wave. When reaching the center of the heated spherical object, the converging spherical wave becomes a diverging spherical rarefaction wave, yielding the late-arriving negative pressure.

\section{DARK-Field CONFocal Photoacoustic Microscopy}

In this section, we introduce the dark-field confocal photoacoustic microscopy (PAM) (Fig. 2) based on a scanning-focused ultrasonic transducer [36], [37]. In conventional dark-field transmission optical microscopy, an opaque disc is placed between the light source and the condenser lens, so that ballistic light is rejected; as a result, only nonballistic light-which is scattered by the sample-is detected. In dark-field PAM, the excitation laser beam has a donut-shaped cross section; therefore, the photoacoustic signal from the tissue surface in the field of view is minimized. The PAM can image optical-absorption contrast beyond the existing depth limit for high-resolution optical imaging; it can achieve a ratio of maximum imaging depth-to-depth resolution (uninterpolated pixel count) greater than 100, which is defined here as high relative spatial resolution.

Acoustic coupling requires contact measurement of the sample. The components within the dashed-box in the photograph (Fig. 2) are translated in a water bath. A window at the bottom of the water container is sealed with an optically and ultrasonically transparent disposable polyethylene membrane (thickness: $44 \mu \mathrm{m})$. After commercial ultrasound gel is applied to the region of interest on the sample for acoustic coupling, the sample is placed between the water container and the sample supporter 
(a)

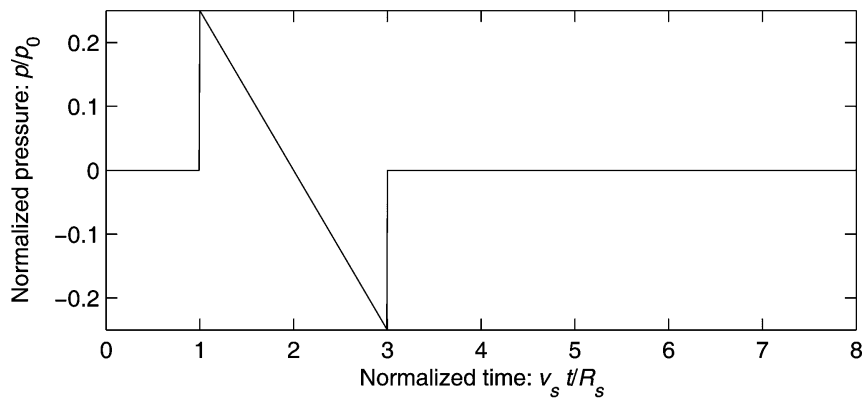

(b)

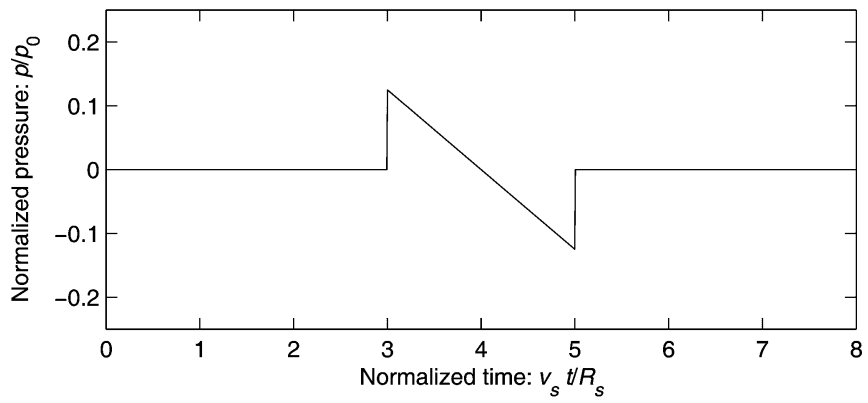

(c)

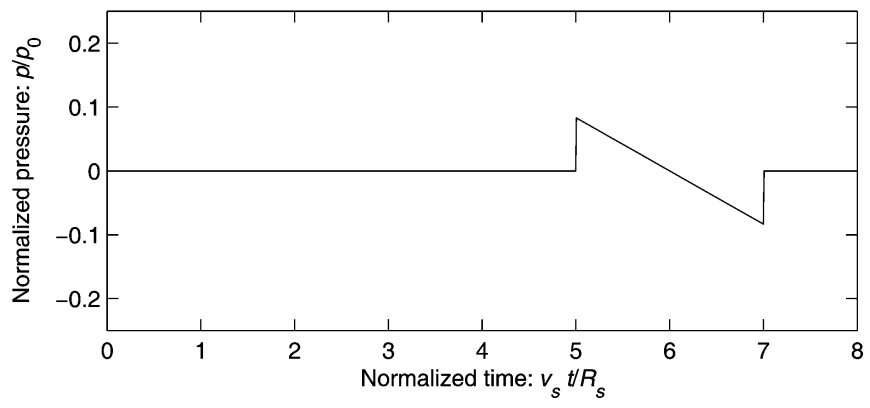

Fig. 1. Bipolar (positive followed by negative) pressure profiles from a homogeneously heated sphere versus time observed at various radial positions [(a) $2 R_{S}$, (b) $4 R_{S}$, and (c) $6 R_{S}$ ] from the center of the sphere. Note the amplitude and duration of each bipolar pulse.

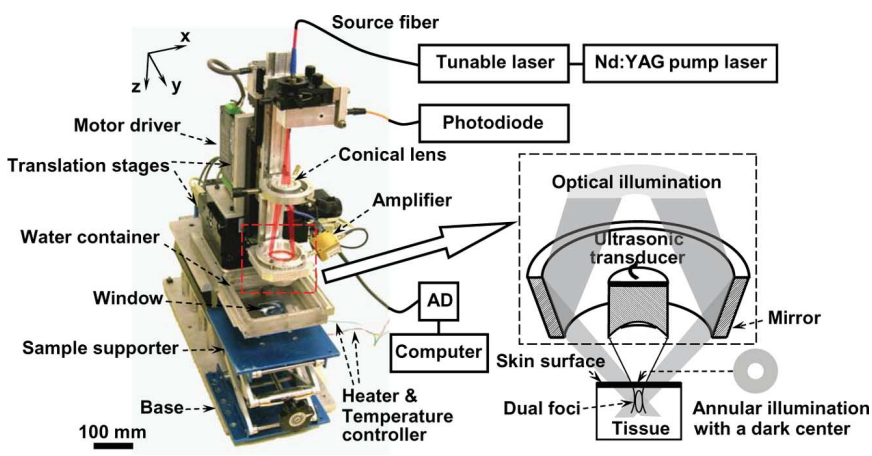

Fig. 2. Photoacoustic microscopy (PAM) system. Reproduced with permission from [37].

for imaging. Note that the sample here can also refer to live subjects.

Image formation starts from the laser. For the generation of photoacoustic waves, $6.5 \mathrm{~ns}$ laser pulses from a tunable dye laser that is pumped by an Nd:YAG laser are used. Such short laser pulses are able to generate photoacoustic waves of a $>100 \mathrm{MHz}$ bandwidth (approximately the reciprocal of the laser pulse width) at the target. Laser light is delivered through an optical fiber to the PAM scanner. Because the optical fiber homogenizes the light beam, no additional measure needs to be taken to avoid hot spots in the laser beam. For hemoglobin imaging using PAM, the laser wavelength is typically set in the Q-band of hemoglobin $(\sim 560 \mathrm{~nm})$ to achieve good SNR. The energy of each laser pulse is recorded by a photodiode for shot-to-shot calibration. The laser beam from the fiber passes through a conical lens to form a ring-shaped illumination pattern. It is then weakly focused into the tissue with the focal region coaxially overlapping the ultrasonic focus inside the tissue. The optical illumination on the skin surface is donut-shaped with a dark center. In an optically clear medium, the optical focus is measured to be $2 \mathrm{~mm}$ in diameter, which is much wider than the ultrasonic focus. The time-resolved photoacoustic wave is recorded at each location of the ultrasonic transducer for $2 \mu \mathrm{s}$, and subsequently, converted into a 1-D depth-resolved image (A-line) based on the sound velocity in soft tissue $(1.54 \mathrm{~mm} / \mu \mathrm{s})$. Then, raster scanning of the dual optical ultrasonic foci in the horizontal $(x-y)$ plane with a step size of $50 \mu \mathrm{m}$ produces a 3-D image. No signal averaging is required in data acquisition for this particular system.

Spatial resolution is determined by the ultrasonic parameters. The lateral resolution of PAM is determined by the focal diameter of the ultrasonic transducer at the center frequency [38], whereas the axial resolution is inversely related to the bandwidth of the ultrasonic transducer. The lateral resolution is usually estimated by the peak-to-zero width of the diffraction pattern on the focal plane, and is given by $0.61\left(\lambda_{0} / \mathrm{NA}\right)$, where $\lambda_{0}$ is the center acoustic wavelength and NA is the numerical aperture of the ultrasonic transducer. Therefore, achieving high spatial resolution requires the use of an ultrasonic transducer with a large NA, a high center frequency, and a wide bandwidth. In the PAM reported here, the ultrasonic detector (V214-BB-RM, Panametrics, WA; element diameter: $6 \mathrm{~mm}$ ) has a $50 \mathrm{MHz}$ center frequency and a $35 \mathrm{MHz}$ nominal bandwidth. A spherically focusing lens provides an NA of 0.44 , a focal length of $6.7 \mathrm{~mm}$, and a focal zone of $0.3 \mathrm{~mm}$. This PAM system is found to have an axial resolution of $\sim 15 \mu \mathrm{m}$ and a lateral resolution of $\sim 45 \mu \mathrm{m}$. An imaging depth of more than $3 \mathrm{~mm}$ in biological tissue has been reached in animals. Therefore, this PAM system has a depth-to-resolution ratio of 200 and is considered to possess high relative spatial resolution. In a soft tissue, the ultrasonic penetration limit is inversely proportional to the ultrasonic frequency because the acoustic attenuation coefficient is proportional to the ultrasonic frequency. As a result, both the spatial resolution and the maximum imaging depth of PAM are scaleable with ultrasonic parameters within the reach of excitation photons.

The major optical absorbers in a biological tissue in the visible spectral region include deoxyhemoglobin (HbR), oxyhemoglobin $\left(\mathrm{HbO}_{2}\right)$, and melanin. At the $584 \mathrm{~nm}$ optical wavelength, PAM can image the morphological relationship between a melanoma tumor and the surrounding blood vessels in the $x-y$ plane, because both melanin and hemoglobin have comparably 


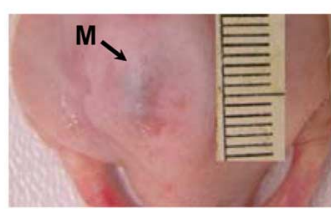

(a)

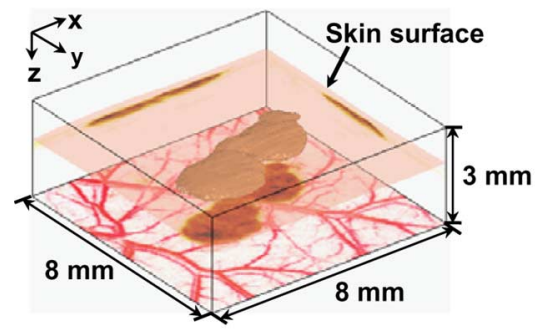

(c)

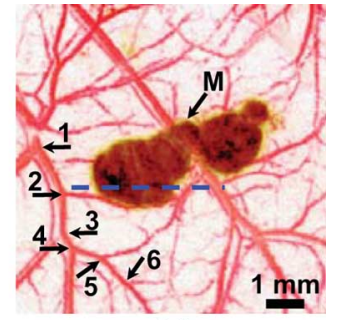

(b)

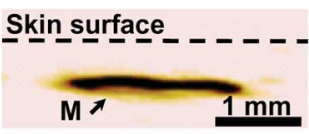

(d)

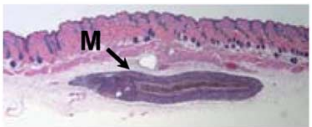

(e)

Fig. 3. Images of a subcutaneously inoculated B16-melanoma in a nude mouse acquired in vivo by PAM at 584 and $764 \mathrm{~nm}$, respectively. (a) Photograph of a melanoma. It shows no tumor morphology or vessel structure. (b) Composite of the two maximum amplitude projections (MAP) images projected along the $z$ axis. Six orders of vessel branching (No. 1-6) can be observed. (c) 3-D rendering of the melanoma acquired at the $764 \mathrm{~nm}$ wavelength. Two MAP images at this wavelength projected along the $x$ - and $y$-axes are shown on the sidewalls. The composite image in panel $b$ is redrawn at the bottom. The top of the tumor is $0.32 \mathrm{~mm}$ below the skin surface and the thickness of the tumor is $0.3 \mathrm{~mm}$. (d) Close-up B-scan image of the melanoma parallel with the $z-x$ plane at the location marked with a dashed line in panel $b$. (e) HE-stained section at the same marked location. M, melanoma. Reproduced with permission from [37].

strong absorption at this wavelength. At the $584 \mathrm{~nm}$ isosbestic optical wavelength, where the two forms of hemoglobin have an identical molar extinction coefficient, the contrast of PAM is sensitive to the total hemoglobin concentration but insensitive to the hemoglobin oxygenation. However, visible light at this wavelength is unsuitable for measuring the tumor thickness because it cannot easily penetrate through the melanin-rich tumor. To overcome this limitation, we form another image using nearinfrared light at the $764 \mathrm{~nm}$ wavelength, where light can infiltrate the tumor because of both the decreased optical absorption of melanin and the minimal absorption of blood. The combination of the two images from these two spectral regions reveals the 3-D morphology of both the melanoma and the surrounding vasculature, where some parallel arterioles and venules are evident (Fig. 3). In the PAM images, the blood vessels from the 584-nm image are shown in red and the melanoma from the $764 \mathrm{~nm}$ image is shown in brown. In Fig. 3(b), microvessels with diameters of less than a single pixel $(50 \mu \mathrm{m})$, presumably resulting from angiogenesis, are observed clearly surrounding the tumor. At the $584 \mathrm{~nm}$ wavelength, the average ratio of the vessel to the background in photoacoustic signal amplitude is $13 \pm 0.89$, and the average ratio of the melanoma tumor to the surrounding blood vessels is $0.92 \pm 0.02$. However, at the $764 \mathrm{~nm}$ wavelength, the average ratio of the melanoma tumor to the blood vessels is $29 \pm 3$, and the average ratio of the melanoma tumor to the background is as high as $68 \pm 5$. Such high optical contrast and specificity are due to the minimal background absorption, and the strong absorption of both hemoglobin and melanin. The average contrast-to-noise ratio

for the melanoma is 67 and $64 \mathrm{~dB}$ at the 584 and $764 \mathrm{~nm}$ wavelengths, respectively. In addition to the functional imaging of total hemoglobin concentration, PAM is capable of providing functional imaging of oxygen saturation of hemoglobin using multiwavelength measurements.

PAM is safe for human subjects since it delivers a transient fluence of only $\sim 6 \mathrm{~mJ} / \mathrm{cm}^{2}$ at the optical focus (pulse energy: $0.2 \mathrm{~mJ}$; focal diameter: $\sim 2 \mathrm{~mm}$ ), which is well within the American National Standard Institute (ANSI) safety standard $\left(20 \mathrm{~mJ} / \mathrm{cm}^{2}\right)$ in the visible spectrum $(400-700 \mathrm{~nm})$ [39]. If a point on the skin is exposed to laser light for more than $10 \mathrm{~s}$, ANSI stipulates that the mean irradiance should not exceed $200 \mathrm{~mW} / \mathrm{cm}^{2}$, which results in a maximum permissible repetition rate of $\sim 30 \mathrm{~Hz}$ here. Since PAM works in raster scanning mode, the exposure duration of any given point on the skin surface is typically less than $10 \mathrm{~s}$. In this case, ANSI requires that the maximum permissible exposure is limited by $1100 t_{e}^{1 / 4}$ in megajoules per centimeter cube, where $t_{e}$ denotes the exposure duration in seconds. This limit translates into $E \times \sqrt[4]{\mathrm{PRF}} \leq 2.75 \times 10^{2} \pi d^{5 / 4} \sigma^{3 / 4}$, where $E$ denotes the pulse energy in megajoules, PRF denotes the pulse repetition frequency in hertz, $d$ denotes the diameter of the illumination area at the ultrasonic focus in centimeter, and $\sigma$ denotes the scanning step size in centimeter [40]. From the parameters of the current PAM, PRF can be as high as $13 \mathrm{kHz}$. The subcutaneous microvasculature of a human palm has been imaged by PAM in vivo (results not shown). The largest vessel in the image is approximately $350 \mu \mathrm{m}$ in diameter, whereas the smallest is within one pixel $(50 \mu \mathrm{m})$.

PAM is fundamentally a high-speed technology. The acquisition time of PAM is currently limited by the $10 \mathrm{~Hz}$ laser pulse repetition frequency. While scanning for a single B-scan image takes only $10 \mathrm{~s}$, a 2-D scan over an $8 \mathrm{~mm} \times 8 \mathrm{~mm}$ area takes more than $18 \mathrm{~min}$ for single-wavelength imaging. The data acquisition can be shortened considerably, however. Each A-line is acquired within only $2 \mu \mathrm{s}$, which equals the maximum imaging depth $(3 \mathrm{~mm})$ divided by the speed of sound $(\sim 1.5 \mathrm{~mm} / \mu \mathrm{s})$. The time lapse between two consecutive laser pulses should be long enough for the photoacoustic wave induced by the first one to subside. If the time lapse is set to $20 \mu \mathrm{s}$, for example, the laser pulse repetition rate can be as high as $50 \mathrm{kHz}$, which is greater than the aforementioned $13 \mathrm{kHz}$ limited by the ANSI safety standard. The author's laboratory is working on accelerating data acquisition using a much higher laser pulse repetition frequency $(2 \mathrm{kHz})$.

\section{Photoacoustic Computed TOMOgRAPHY}

In this section, we consider the reconstruction-based photoacoustic computed tomography (PAT) that is formed by scanning an unfocused ultrasonic transducer-ideally a point transducer-followed by image reconstruction. The initial photoacoustic pressure excited by laser pulse $\delta(t)$ equals $p_{0}(\mathbf{r})$ [see (8)]. The acoustic pressure $p\left(\mathbf{r}_{0}, t\right)$ at position $\mathbf{r}_{0}$ and time $t$, initiated by source $p_{0}(\mathbf{r})$, is measured around the tissue by an unfocused ultrasonic transducer.

Spherical, cylindrical, and planar detection configurations are considered (Fig. 4), where the detection surface $S_{0}$ encloses the 


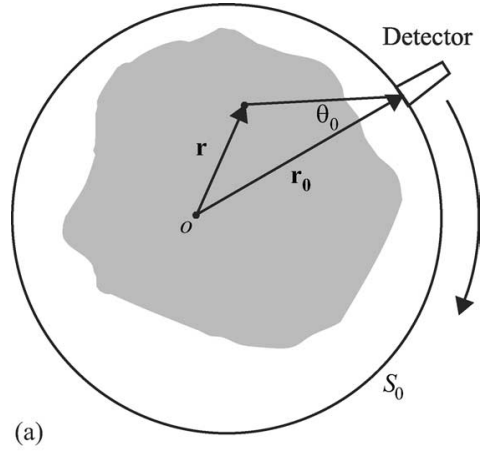

(b)

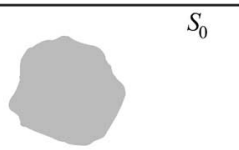

Fig. 4. Reconstruction-based photoacoustic computed tomography. (a) Spherical or cylindrical detection configuration. During measurement, an ultrasonic point detector at position $\mathbf{r}_{0}$ on surface $S_{0}$ receives photoacoustic signals emitted from source $p_{0}(\mathbf{r})$. During image reconstruction, a quantity related to the measurement at position $\mathbf{r}_{0}$ projects backward via a spherical surface (not shown) centered at $\mathbf{r}_{0}$. (b) Planar detection configuration.

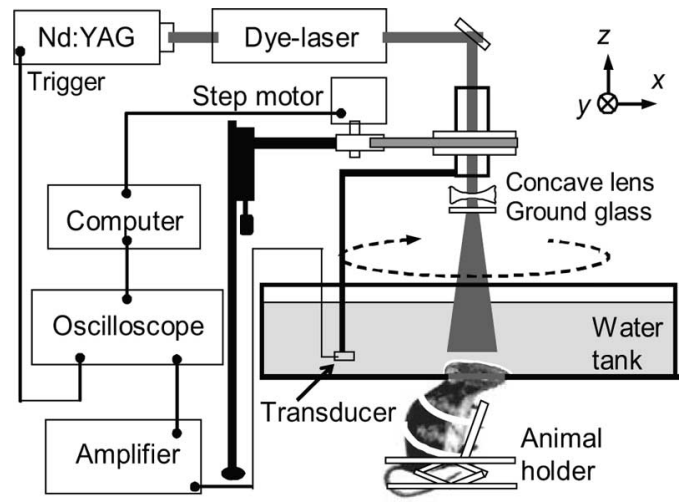

Fig. 5. Diagram of a circular-scanning PAT system for small-animal imaging. Reproduced with permission from [43].

source $p_{0}(\mathbf{r})$. For brevity, we simply provide the final backprojection formula [41] without showing the derivation

$$
p_{0}(\mathbf{r})=\frac{1}{\Omega_{0}} \int_{S_{0}}\left[2 p\left(\mathbf{r}_{0}, t\right)-\frac{2 t \partial p\left(\mathbf{r}_{0}, t\right)}{\partial t}\right] \frac{\cos \theta_{0}}{\left|\mathbf{r}-\mathbf{r}_{0}\right|^{2}} d S_{0}
$$

where $\Omega_{0}=2 \pi$ for the planar geometry, $\Omega_{0}=4 \pi$ for the spherical or cylindrical geometry, and $\theta_{0}$ denotes the angle between the detection surface normal and the vector pointing to the reconstruction point $\mathbf{r}$, as illustrated in Fig. 4(a).

A representative PAT system [42], [43] is shown in Fig. 5. An Nd:YAG laser (Brilliant B, Bigsky), operating at a wavelength of $532 \mathrm{~nm}$ with a pulsewidth of $6.5 \mathrm{~ns}$ and a pulse repetition rate of $10 \mathrm{~Hz}$, pumps a tunable dye laser (ND6000, Continuum). A small animal (rat or mouse) to be imaged is immobilized on an animal holder. The head of the animal protrudes into a water tank through an opening at the bottom of the tank, where the opening is sealed using a clear polyethylene membrane. The laser beam is expanded and homogenized to provide relatively uniform incident fluence, which is less than $10 \mathrm{~mJ} / \mathrm{cm}^{2}$ on

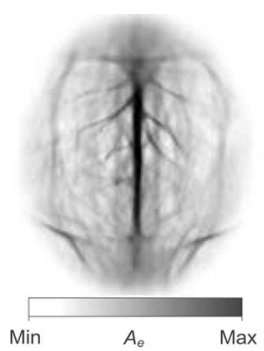

(a)

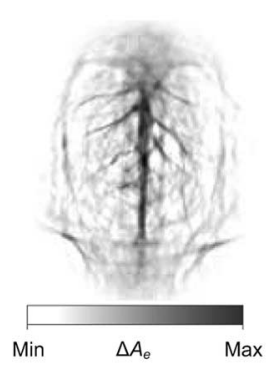

(c)

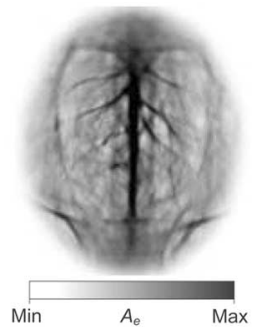

(b)

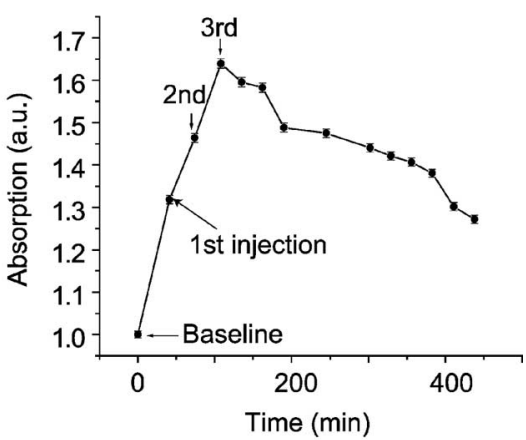

(d)
Fig. 6. In vivo PAT images of a rat brain acquired. (a) Before three sequential administrations of nanoshells. (b) $\sim 20$ min after three sequential administrations of nanoshells. (c) Differential image $(c=b-a)$. Field of view: $20 \times 20 \mathrm{~mm}$. Ae, optical absorption; $\Delta A_{e}$, differential optical absorption. (d) Differential signal versus time. Adapted with permission from [43].

the skin surface to conform to the ANSI standards [39]. Upon laser excitation, photoacoustic waves are produced in the head. An unfocused ultrasonic transducer (XMS-310, Panametrics) with a central frequency of $10.4 \mathrm{MHz}$, a bandwidth of $100 \%$ at $\sim 6 \mathrm{~dB}$ and an active element of $2 \mathrm{~mm}$ in diameter is submerged in water to detect the photoacoustic signals. A rotational detection system that is driven by a computer-controlled stepper motor scans the transducer around the brain with a radius of $3 \mathrm{~cm}$ and a step size of $1.5^{\circ}$. The data acquisition time to obtain a single 2-D image slice is $\sim 24 \mathrm{~min}$ at this pulse repetition rate. The photoacoustic signals detected by the transducer are amplified by an amplifier, and then, digitized by an oscilloscope (TDS 540B, Tektronix). Finally, a computer acquires the signals for image reconstruction.

Since a circle - rather than a full spherical surface-is usually scanned, the reconstruction algorithm given by (28) is only approximately applicable. Nevertheless, good images are still attainable. The in-plane spatial resolution of this imaging system, limited mainly by the bandwidth of the detected photoacoustic signals, is $\sim 60 \mu \mathrm{m}$. The out-of-plane resolution, limited by the aperture effect of the transducer, is $\sim 2 \mathrm{~mm}$. Alternatively, ultrasonic transducer cylindrically focused in the direction perpendicular to the imaging plane can be used to improve the out-of-plane resolution. In addition, a cylindrical scan can be taken to form a 3-D image with better out-of-plane resolution.

We demonstrate the imaging of nanoshells (silica core diameter: $125 \pm 5 \mathrm{~nm}$; gold shell thickness: $10-12 \mathrm{~nm}$ ), which serve as a contrast agent for PAT, in a rat in vivo [43]. Two of the PAT images of the cerebral cortex of the rat brain are presented in Fig. 6(a) and (b), where the color bars are identical. With the 
TABLE I

COMPARISON OF High-RESOLUTION OPTICAL IMAGING MODALITIES

\begin{tabular}{|l|l|l|}
\hline Modality & Penetration & Primary contrast \\
\hline Confocal microscopy & $\sim 0.5 \mathrm{~mm}$ & Scattering, fluorescence \\
\hline Two-photon microscopy & $\sim 0.5 \mathrm{~mm}$ & Fluorescence \\
\hline Optical coherence tomography & $\sim 1 \mathrm{~mm}$ & Scattering, polarization \\
\hline PAI & $\sim 3-30 \mathrm{~mm}$, scalable & Absorption \\
\hline
\end{tabular}

exogenous contrast agent in the blood, the optical absorption of the blood at $800 \mathrm{~nm}$ was increased, and the contrast between the vessels and the background brain tissues was enhanced. The differential image in Fig. 6(c) depicts the distribution of differential optical absorption in the rat brain due to the exogenous contrast agent. The optical absorptions in the major blood vessels were averaged to represent the relative concentration of nanoshells in the circulatory system of the rat [Fig. 6(d)]. After each of the three administrations, the optical absorption of blood increased. The first administration increased the absorption of blood by $\sim 30 \%$, whereas the three administrations together increased the absorption by $\sim 63 \%$.

Then, the optical absorption decreased owing to clearance of the nanoshells from the blood.

After the first administration of the nanoshells, the concentration of nanoshells in the blood is estimated to be $\sim 10^{10}$ nanoshells/mL. Since the in-plane spatial resolution of this PAT system is $\sim 60 \mu \mathrm{m}$ and the diameters of the cortical blood vessels under study are $<100 \mu \mathrm{m}$, the number of nanoshells in the $60 \mu \mathrm{m} \times 60 \mu \mathrm{m} \times 100 \mu \mathrm{m}$ resolvable volume is $\sim 3600$, which represents an estimate of the sensitivity of this PAT system to nanoshells.

\section{COMPARISON With OTHER HIGH-RESOLUTION OPTICAL IMAGING MODALITIES}

Although several commercially available pure optical imaging modalities, including confocal microscopy, two-photon microscopy, and optical coherence tomography, have been highly successful, none of these technologies can provide penetration beyond $\sim 1 \mathrm{~mm}$ into scattering biological tissues because they are based on ballistic and quasi-ballistic photons. Heretofore, there has been a void in the high-resolution optical imaging beyond this penetration limit. Photoacoustic tomography, which combines high ultrasonic resolution and strong optical contrast in a single modality, has broken through this penetration limitation and filled this void. Table I compares the various highresolution optical imaging modalities. The various modalities complement each other in both penetration and contrast, and therefore, will coexist. While photoacoustic imaging is sensitive to optical absorption, it is insensitive to optical scattering or fluorescence.

Photoacoustic imaging is more sensitive to optical absorption than that of the other modalities. Since $A_{e}=\mu_{a} F$, where $\mu_{a}$ denotes the optical absorption coefficient and $F$ denotes the optical fluence (in joules per centimeter square), (8) becomes

$$
p_{0}=\Gamma \mu_{a} F \text {. }
$$

Hence, the photoacoustic signal $\left(p_{0}\right)$ is proportional to the optical absorption coefficient. If we focus on the variation in the local absorption coefficient, we have $\Delta p_{0} / p_{0}=\Delta \mu_{a} / \mu_{a}$, where $\Delta$ indicates a small variation in the modified variable. Here, the effect of $\Delta \mu_{a}$ on $\Delta p_{0}$ through $F$ is neglected. Therefore, any fractional change in the optical absorption coefficient translates into an equal amount of fractional change in the photoacoustic signal, which means a relative sensitivity of unity.

By contrast, the signal in confocal microscopy can be modeled as

$$
I_{\mathrm{cf}} \propto R_{b} \exp \left[-2\left(\mu_{a}+\mu_{s}\right) z_{p}\right]
$$

where $R_{b}$ denotes the percentage of the back-scattered light to be received by the detector, $\mu_{s}$ denotes the optical scattering coefficient, $z_{p}$ denotes the length of a pixel, and the factor 2 indicates the effect of the round-trip ballistic light propagation. The effect of the variation in the absorption coefficient can be expressed as

$$
\Delta I_{\mathrm{cf}} / I_{\mathrm{cf}}=\frac{\left(-2 \mu_{a} z_{p}\right) \Delta \mu_{a}}{\mu_{a}} .
$$

For typical pixel length $z_{p}=1 \mu \mathrm{m}$ and hemoglobin absorption coefficient $\mu_{a}=4,300$, and $3000 \mathrm{~cm}^{-1}$ at 800,560 (Q-band), and $420 \mathrm{~nm}$ (Soret band), respectively, the sensitivities $S_{\mathrm{cf}}=2 \mu_{a} z_{p}$ are $8 \times 10^{-4}, 6 \times 10^{-2}, 6 \times 10^{-1}$, respectively. Unless the Soret band is chosen, the sensitivity to optical absorption of confocal microscopy is significantly lower than that of photoacoustic imaging. However, the operation at the Soret band limits the penetration severely because the penetration depth is $\sim 3 \mu \mathrm{m}$ in blood.

In comparison to confocal microscopy, two-photon microscopy has half the sensitivity to the one-photon optical absorption coefficient because the two-photon excitation involves only one-way ballistic optical propagation and the two-photon fluorescence detection can tolerate multiple scattering. Interestingly, optical coherence tomography also has half the sensitivity because the electric field instead of the intensity is detected interferometrically. As a result, the sensitivity becomes $4 \times 10^{-3}$ around the typically used $800 \mathrm{~nm}$ wavelength with $z_{p}=10 \mu \mathrm{m}$.

\section{Limitations OF PHOtOACOUSTIC IMAGING}

There are two important depth penetration limits for optical imaging. The $1 \mathrm{~mm}$ optical penetration limit is approximately correlated with the optical transport mean free path, representing the depth of the quasi-ballistic regime in biological tissue [32]. We refer to this limit as the soft limit for high-resolution optical imaging. Photoacoustic imaging is able to break through this soft limit. Another limit exists at $\sim 50 \mathrm{~mm}$ depth, which equals roughly ten times the 1/e optical penetration depth in the nearinfrared window ( $\sim 700-800 \mathrm{~nm}$ wavelength). Penetration to this limit in the diffusive regime requires approximately $43 \mathrm{~dB}$ one-way energy decay. We refer to this limit as the hard limit for optical imaging although it does not represent a theoretical one. Beyond this limit, the optical energy is low and even diffuse photons are few. Diffuse optical tomography and photoacoustic tomography have reached this limit [44], [45]. However, if we 
image tissue such as the human breast from both sides, a $10 \mathrm{~cm}$ thickness can be potentially imaged, which is adequate for many biomedical applications. One approach to overcome the optical penetration limitation is to adopt microwaves for photoacoustic excitation [5], [6]. In this case, the technology is referred to as microwave-induced acoustic (thermoacoustic) imaging.

\section{SUMMARY AND DisCUSSION}

Several differences between the earlier described two representative implementations of photoacoustic imaging are summarized here. First, the confocal PAM operates in reflection (backward) mode, whereas the reconstruction-based PAT works in orthogonal mode. Second, the confocal PAM provides lateral resolution using spherical focusing of the ultrasonic transducer and the axial resolution using the time resolution of the transducer. In contrast, the circular-scanning PAT derives in-plane resolution from reconstruction and out-of-plane resolution from the aperture effect or cylindrical focusing of the ultrasonic transducer. To some extent, the reconstruction can be thought of as a numerical acoustic lens, or the acoustic lens can be thought of as hardware reconstruction. Third, the confocal PAM is based on a scanning single-element ultrasonic transducer, whereas the reconstruction-based PAT can be implemented with an array of ultrasonic transducers for faster data acquisition. It should be mentioned that the photoacoustic imaging can be implemented in various other forms depending on the applications in mind.

Common to both PAM and PAT, the pulsewidth of the laser must be carefully chosen. As demonstrated with the PAM system, $3 \mathrm{~mm}$ penetration with a depth-to-resolution ratio more than 100 can be achieved at $50 \mathrm{MHz}$. To produce photoacoustic signals around this frequency, one should choose a laser with a pulsewidth less than $1 / 50 \mu$ s or 20 ns. A Q-switched laser satisfies this requirement. Mode-locked lasers can produce pulses of picosecond or femtosecond durations at usually lower pulse energy. Such short durations are unnecessary for the $3 \mathrm{~mm}$ penetration system but can be useful for smaller penetration and higher resolution systems.

The spectral range of the laser must be carefully selected as well. For a $3 \mathrm{~mm}$ penetration, the Q-band $(\sim 560 \mathrm{~nm})$ of hemoglobin is ideal for imaging of hemoglobin because of the moderately high optical absorption of hemoglobin in this spectral region. The high optical absorption provides strong photoacoustic signals and high signal-to-background ratio while the ANSI safety limit is satisfied. At the same time, the optical penetration is sufficient to reach a few millimeters depth. For deeper (e.g., $30 \mathrm{~mm}$ ) penetration, the near-infrared window around 700-800 nm should be used instead. However, the photoacoustic signal becomes lower and typically requires averaging to enhance the SNR.

In summary, PAI has the following capabilities. 1) PAI breaks through the current fundamental depth limit of high-resolution optical imaging modalities while maintaining the depth-toresolution ratio greater than 100.2$)$ The depth-to-resolution ratio remains approximately constant while the imaging depth and spatial resolution are scaled. On the one hand, the imaging depth can be scaled up to potentially centimeters, which is limited by the penetration depth of diffuse light rather than ballistic light. On the other hand, the spatial resolution can be scaled down to $<10 \mu \mathrm{m}$, where the imaging depth is limited by the penetration depth of high-frequency ultrasound. 3) PAI provides high sensitivity to optical absorption. 4) PAI provides functional imaging based on physiologically specific endogenous optical absorption contrasts. 5) PAI provides molecular or reporter gene imaging based on exogenous contrasts. 6) PAI can potentially be constructed to image in real time. 7) PAI provides speckle-free images. 8) PAI is safe for human subjects, and it is ready for clinical application.

\section{REFERENCES}

[1] A. G. Bell, "On the production and reproduction of sound by light," Amer. J. Sci., vol. 20, pp. 305-324, 1880.

[2] A. A. Karabutov, N. B. Podymova, and V. S. Letokhov, "Time-resolved laser optoacoustic tomography of inhomogeneous media," Appl. Phys. B-Lasers Opt., vol. 63, pp. 545-563, 1996.

[3] C. G. A. Hoelen, F. F. M. de Mul, R. Pongers, and A. Dekker, "Threedimensional photoacoustic imaging of blood vessels in tissue," Opt. Lett., vol. 23, pp. 648-650, 1998.

[4] R. O. Esenaliev, A. A. Karabutov, and A. A. Oraevsky, "Sensitivity of laser opto-acoustic imaging in detection of small deeply embedded tumors," IEEE J. Sel. Topics Quantum Electron., vol. 5, no. 4, pp. 981-988, Jul./Aug. 1999.

[5] R. A. Kruger, D. R. Reinecke, and G. A. Kruger, "Thermoacoustic computed tomography-Technical considerations," Med. Phys., vol. 26, pp. 1832-1837, 1999.

[6] L.-H. V. Wang, X. Zhao, H. Sun, and G. Ku, "Microwave-induced acoustic imaging of biological tissues," Rev. Sci. Instrum., vol. 70, pp. 3744-3748, 1999.

[7] A. A. Karabutov, E. V. Savateeva, N. B. Podymova, and A. A. Oraevsky, "Backward mode detection of laser-induced wide-band ultrasonic transients with optoacoustic transducer," J. Appl. Phys., vol. 87, pp. 2003$2014,2000$.

[8] G. Ku and L.-H. V. Wang, "Scanning microwave-induced thermoacoustic tomography: Signal, resolution, and contrast," Med. Phys., vol. 28, pp. 4 10, 2001.

[9] K. P. Kostli, D. Frauchiger, J. J. Niederhauser, G. Paltauf, H. P. Weber, and M. Frenz, "Optoacoustic imaging using a three-dimensional reconstruction algorithm," IEEE J. Sel. Topics Quantum Electron., vol. 7, no. 6, pp. 918-923, Nov./Dec. 2001.

[10] K. P. Kostli, M. Frenz, H. P. Weber, G. Paltauf, and H. Schmidt-Kloiber, "Optoacoustic tomography: Time-gated measurement of pressure distributions and image reconstruction," Appl. Opt., vol. 40, pp. 3800-3809, 2001.

[11] G. Ku and L.-H. V. Wang, "Scanning microwave-induced thermoacoustic tomography: Signal, resolution, and contrast," Med.Phys., vol. 28, pp. 410,2001

[12] M. H. Xu, G. Ku, and L.-H. V. Wang, "Microwave-induced thermoacoustic tomography using multi-sector scanning," Med.Phys., vol. 28, pp. 19581963, 2001.

[13] Y. Xu and L.-H. V. Wang, "Signal processing in scanning thermoacoustic tomography in biological tissues," Med. Phys., vol. 28, pp. 1519-1524, 2001.

[14] G. Paltauf, J. A. Viator, S. A. Prahl, and S. L. Jacques, "Iterative reconstruction algorithm for optoacoustic imaging," J. Acoust. Soc. Amer., vol. 112, pp. 1536-1544, 2002.

[15] M. H. Xu and L.-H. V. Wang, "Time-domain reconstruction for thermoacoustic tomography in a spherical geometry," IEEE Trans. Med. Imag., vol. 21, no. 7, pp. 814-822, Jul. 2002.

[16] Y. Xu, D. Z. Feng, and L.-H. V. Wang, "Exact frequency-domain reconstruction for thermoacoustic tomography-I: Planar geometry," IEEE Trans. Med. Imag., vol. 21, no. 7, pp. 823-828, Jul. 2002.

[17] Y. Xu, M. H. Xu, and L.-H. V. Wang, "Exact frequency-domain reconstruction for thermoacoustic tomography-II: Cylindrical geometry," IEEE Trans. Med. Imag., vol. 21, no. 7, pp. 829-833, Jul. 2002.

[18] V. G. Andreev, A. A. Karabutov, and A. A. Oraevsky, "Detection of ultrawide-band ultrasound pulses in optoacoustic tomography," IEEE Trans. Ultrason., Ferroelectr., Freq. Control, vol. 50, no. 10, pp. 13831390, Oct. 2003. 
[19] D. Finch, S. K. Patch, and R. Rakesh, "Determining a function from its mean values over a family of spheres," Siam J. Math. Anal., vol. 35, pp. 1213-1240, 2003.

[20] A. A. Karabutov, E. V. Savateeva, and A. A. Oraevsky, "Optoacoustic tomography: New modality of laser diagnostic systems," Laser Phys., vol. 13, pp. 711-723, 2003.

[21] K. P. Kostli and P. C. Beard, "Two-dimensional photoacoustic imaging by use of Fourier-transform image reconstruction and a detector with an anisotropic response," Appl. Opt., vol. 42, pp. 1899-1908, 2003.

[22] X. D. Wang, Y. J. Pang, G. Ku, G. Stoica, and L.-H. V. Wang, "Threedimensional laser-induced photoacoustic tomography of mouse brain with the skin and skull intact," Opt. Lett., vol. 28, pp. 1739-1741, 2003.

[23] M. H. Xu and L.-H. V. Wang, "Analytic explanation of spatial resolution related to bandwidth and detector aperture size in thermoacoustic or photoacoustic reconstruction," Phys. Rev. E, vol. 67, pp. 056605-1056605-15, 2003.

[24] J. A. Copland, M. Eghtedari, V. L. Popov, N. Kotov, N. Mamedova, M. Motamedi, and A. A. Oraevsky, "Bioconjugated gold nanoparticles as a molecular based contrast agent: Implications for imaging of deep tumors using optoacoustic tomography," Mol. Imag. Biol., vol. 6, pp. 341-349, 2004.

[25] M. Haltmeier, O. Scherzer, P. Burgholzer, and G. Paltauf, "Thermoacoustic computed tomography with large planar receivers," Inverse Probl., vol. 20, pp. 1663-1673, 2004.

[26] G. Ku, X. D. Wang, G. Stoica, and L.-H. V. Wang, "Multiple-bandwidth photoacoustic tomography," Phys. Med. Biol., vol. 49, pp. 1329-1338, 2004.

[27] Y. Xu and L.-H. V. Wang, "Time reversal and its application to tomography with diffracting sources," Phys. Rev. Lett., vol. 92, pp. 033902-1-0339024, 2004.

[28] B. T. Cox and P. C. Beard, "Fast calculation of pulsed photoacoustic fields in fluids using k-space methods," J. Acoust. Soc. Amer., vol. 117, pp. 3616-3627, 2005

[29] G. Ku and L.-H. V. Wang, "Deeply penetrating photoacoustic tomography in biological tissues enhanced with an optical contrast agent," Opt. Lett., vol. 30, pp. 507-509, 2005.

[30] G. Ku, X. D. Wang, X. Y. Xie, G. Stoica, and L.-H. V. Wang, "Imaging of tumor angiogenesis in rat brains in vivo by photoacoustic tomography," Appl. Opt., vol. 44, pp. 770-775, 2005.

[31] J. Zhang, M. A. Anastasio, X. C. Pan, and L.-H. V. Wang, "Weighted expectation maximization reconstruction algorithms for thermoacoustic tomography," IEEE Trans. Med. Imag., vol. 24, no. 6, pp. 817-820, Jun. 2005.

[32] L.-H. V. Wang and H. Wu, Biomedical Optics: Principles and Imaging. Hoboken, NJ: Wiley, 2007.

[33] M. Xu and L.-H. V. Wang, "Photoacoustic imaging in biomedicine," Rev. Sci. Instrum., vol. 77, pp. 041101-1-041101-22, 2006.

[34] V. E. Gusev and A. A. Karabutov, Laser Optoacoustics. New York: American Institute of Physics, 1993.

[35] P. M. Morse and H. Feshbach, Methods of Theoretical Physics. New York: McGraw-Hill, 1953.

[36] K. Maslov, G. Stoica, and L.-H. V. Wang, "In vivo dark-field reflectionmode photoacoustic microscopy," Opt. Lett., vol. 30, pp. 625-627, 2005.

[37] H. F. Zhang, K. Maslov, G. Stoica, and L.-H. V. Wang, "Functional photoacoustic microscopy for high-resolution and noninvasive in vivo imaging," Nat. Biotechnol., vol. 24, pp. 848-851, 2006.
[38] G. A. D. Briggs, Acoustic Microscopy. Oxford, U.K.: Clarendon, 1992.

[39] American National Standard for Safe Use of Lasers, Laser Inst. Amer., ANSI Standard Z136.1-2000, NY, 2000.

[40] H. F. Zhang, K. Maslov, and L.-H. V. Wang, "In vivo imaging of subcutaneous structures using functional photoacoustic microscopy," Nat. Protoc., vol. 2, pp. 797-804, 2007.

[41] M. H. Xu and L.-H. V. Wang, "Universal back-projection algorithm for photoacoustic computed tomography," Phys. Rev. E, vol. 71, pp. 0167061-016706-7, 2005

[42] X. D. Wang, Y. J. Pang, G. Ku, X. Y. Xie, G. Stoica, and L.-H. V. Wang, "Noninvasive laser-induced photoacoustic tomography for structural and functional In vivo imaging of the brain," Nat. Biotechnol., vol. 21, pp. 803806, 2003.

[43] Y. W. Wang, X. Y. Xie, X. D. Wang, G. Ku, K. L. Gill, D. P. O’Neal, G. Stoica, and L.-H. V. Wang, "Photoacoustic tomography of a nanoshell contrast agent in the In vivo rat brain," Nano Lett., vol. 4, pp. 1689-1692, 2004.

[44] G. Ku and L.-H. V. Wang, "Deeply penetrating photoacoustic tomography in biological tissues enhanced with an optical contrast agent," Opt. Lett., vol. 30, pp. 507-509, 2005.

[45] G. Ku, B. D. Fornage, X. Jin, M. H. Xu, K. K. Hunt, and L.-H. V. Wang, "Thermoacoustic and photoacoustic tomography of thick biological tissues toward breast imaging," Technol. Cancer Res. Treat., vol. 4, pp. 559$565,2005$.

Lihong V. Wang (M'96-SM'00-F'06) received the Ph.D. degree in electrical engineering from Rice University, Houston, TX, in 1992.

$\mathrm{He}$ is currently the Gene K. Beare Distinguished Professor in the Department of Biomedical Engineering, Washington University, St. Louis, MO, where he is also the Directer of the Optical Imaging Laboratory. He is the author or coauthor of more than 135 peer-reviewed journal articles published in various international journals. He is the author of the first textbooks in biomedical optics entitled Biomedical Optics: Principles and Imaging. He invented or discovered the dark-field confocal photoacoustic microscopy, photoacoustic Doppler sensing, focused scanning microwave-induced thermoacoustic tomography, exact reconstruction algorithms for photoacoustic or thermoacoustic tomography, frequency-swept ultrasound-modulated optical tomography, sonoluminescence tomography, Mueller-matrix optical coherence tomography, and oblique-incidence reflectometry. His Monte Carlo model of photon transport in scattering media has been used worldwide as a standard tool. He serves on the editorial boards for the Journal of Biomedical Optics and the Applied Optics.

Dr. Wang is the Chair of the International Biomedical Optics Society. He is the recipient of the National Institutes of Health (NIH) FIRST Award, National Science Foundation (NSF) CAREER Award, and the Outstanding Young Scientist Award sponsored by the Johnson \& Johnson Medical, Inc., and the Houston Society for Engineering in Medicine and Biology. He is also a Fellow of the American Institute for Medical and Biological Engineering, the Optical Society of America, and the Society of Photo-Optical Instrumentation Engineers. 